\title{
Reviews of the Study of the Language in Unearthed Warring States and Qin Dynasty Literature
}

\author{
Weiming Peng \\ College of Liberal Arts \\ South China Normal University \\ Guangzhou, China
}

\author{
Yanmei Zhang* \\ School of Foreign Language \\ Guilin University of Electronic Technology \\ Guilin, China \\ *Corresponding Author
}

\author{
Yixin $\mathrm{Hu}$ \\ School of Foreign Language \\ Guilin University of Electronic Technology \\ Guilin, China
}

\begin{abstract}
The research of unearthed literature in warring states period has achieved a series of new developments and abundant outcomes by scholar in recent years. And this article made brief evaluations and prospects from explanations of Chinese characters, vocabulary arrangement, grammar research, interchangeable words and arrangement of corpus indexing tools this five aspects.
\end{abstract}

Keywords-unearthed literature; Warring States; Qin dynasty; review

\section{INTRODUCTION}

Be universally known, it has great scientific value of unearthed literature. And readers could understand the latest ancient Chinese research and realize the significance of subject theory of soil Philology through this article. No only it has practical value of using the Warring States Qin Dynasty unearthed literature to teach and research palaeography, ancient Chinese grammar and Chinese history, but also it has great value of other subject research.

This article mainly introduces the language research status of the Warring States unearthed literature (including bamboo silks, inscriptions on bronze, jade, etc.) that Chinese scholar did during January 2011 to December 2016.The era of unearthed literatures and material were limited by warring states period (269 years).

There are four kinds of the Warring States unearthed literature: (1) The Warring States Qin Dynasty bronze inscription. (2)the Warring States Qin Bamboo character(including the Ceng Houyi tomb bamboo slips, Guodian Chu bamboo slips, Xincai Chu bamboo slips, Xinyang Chu bamboo slips, Wulipai Chu bamboo slips, Yangtian Lake Chu bamboo slips, Yangjiawan Chu bamboo slips, Wangshan Chu bamboo slips, Jiudian Chu bamboo slips, Baoshan Chu bamboo slips, Chinese University Hong Kong Chu bamboo slips, Tsinghua University Chu bamboo slips,Tianxingguan Chu bamboo slips, Shuihudi Qin
Bamboo Slips, and Shuihudi slips, Qingchuan Qin slips,Fong Ma beach Qin bamboo slips, mountain Yue Qin bamboo slips, Longgang Qin bamboo slips, Zhou Jia Tai Qin bamboo slips, Wang Jia Tai Qin bamboo slips, Liye Qin bamboo slips,etc.). (3)The Warring States silk (i.e. Zidanku Changsha Warring States Chu silk).(4)the Warring States Qin Dynasty Jade text (including the Yinyu version of Qi jade seal,Xingqi jade seal, Yuhuangjian jade seal, Shou Qiu stones,Zuchu script, Yi Shan stone, etc.).

The content of the Warring States bamboo character is the most abandon, and with a large number of research outcome. However, this article just introduces parts of it, because of the restriction of article length and learning capacity. Besides, the works that are not mentioned in this article do not represent the author's view that it is unimportant or disagree with it. This is what the author wants readers to understand.

\section{EXPLANATION OF CHINESE CHARACTERS}

The publishment of textual reference book, takes a grand scene.

First of all, the comprehensive text edit were reprinted many times, and got better and better. The achievement of this area includes The Chinese Character Compilation of Warring States Period (Revised Edition)'(Xu Zaiguo, 2015).By contrast to the first edition, it has been adjusted by eight aspects by follow: (1) It was deleted for five editing prefix characters, such as “JIE (莭), 䟜”, etc. (2) It was added for three editing prefix characters, such as “䟜”. (3) It was fine-tuned for one preparing prefix, and that is "DU (妬)". (4) The were 157 mistakes about the character font of edit and combination revised, such as “LI (吏), CI (祠), MANG(莽)", etc. (6) It was adjusted for five editing character fonts, such as “䟜, SHAN (善), GOU (苟)”, etc. (7) It was added for fifteen editing annotation. (8) It was deleted several cases by edition. 
The Chinese Character Compilation of Warring States Period (Revised Edition) not only is a prior academic monograph of summary study for Warring States characters in twenty-first century, but also is a dictionary which can reflect the characters of Warring states period comprehensively. It is a masterpiece of Chinese character explanation in the ancient Chinese dictionary of Warring States after the monograph Ancient Chinese Dictionary of Warring States: The Literary System of the Warring States (He Linyi, 1998) was published.

In addition, it is refreshing about the compilation and the outcome of a series of textual reference books by the publishment from East China Normal University. For example, the textual reference book The Summary Overview of Textual Criticism and Explanation of the Ancient Writing (Volume One to Volume Three), (Liu Zhiji,2011) inherited various opinions which is collected and heckled from separate parts to ancient Chinese characters, especially the character of Warring States from Origin of Chinese Characters. And it quests for perfection constantly, for example, not only it summarizes various opinions, but also proposes its own tendentious views. Besides, the compiling ideas of that book interact with The Explanatory Dictionary of Ancient Chinese Characters, the former is concise and brief and the latter is full and accurate.

Secondly, the compilation of regional writing dictionary has taken on new look. The character of every Warring States series has published writing dictionary basically, except for Yan character material because of few amount and rare material. For example, Qin Writing Dictionary(Wang Hui, 2015), SanJin Writing Dictionary(Yang Zhibiao,2013), Qi Writing Dictionary(Sun Gang, 2010) and Qilu Writing Dictionary(Zhang Zhenqian, 2014).In the future, it is possible to see the writing dictionary of combining every region and many materials. However, the writing dictionary in separate region is less likely to be published, except for Chu series which could be revised Chu Writing Dictionary (Li Shoukui, 2003) along with discovering the unearthed material increasingly.

Thirdly, the compilation of single materials. There are some monographs about researching unearthed materials which are published, such as New Discovered Jin Writing Dictionary (Chen Sipeng,2011),Collection of Tsinghua University Bamboo Slips of Warring States Writing Dictionary I - III (Li xueqin,2014), Tsinghua University Shaped Table of Warring States Bamboo(Volume Four, Five, Six, Seven),(Li Xueqin,etc.2014,2015,2016,2017), Baoshan Chu Tomb Full Text(Li Shoukui,2012), The vocabulary of Chu Bamboo Slips in The Warring States Period in Shanghai Museum(Rao Zongyi,2012), and The Shanghai museum Text Sound Slips I -VIII(Xu zaiguo,2013),etc.

At last, the monograph A Comparative Study of Words and Phrases in the Warring States Period(Zhou bo,2011) must be mentioned because it is a masterpiece that researched the character in Warring States period from the aspect of differences of using characters. This project is based on his doctoral dissertation A Study On the Difference of Characters Used in Different Periods of the Warring States
Period, and carries on deeper research. Of course, there has been no news of the results of the publication of the project, so it is not evaluated here.

\section{VOCABULARY COMPILATION}

The outcome of vocabulary compilation is most flourishing. For example, The Warring States Period to the Early Han Dynasty Unearthed Bamboo Silk See Figure Name Explanations and Research (Wang Hui,2014)and The Warring States and Qin Dynasty's Full Place Names (Wu Liangbao,2014).Wu has always paid attention to the arrangement of place name in Warring States period, especially the place name in Chu. The representative opus about researching the place name on Chu bamboo silk is Chu Kingdom Place Name Cards (Wu Liangbao, 2006, 2010).This monograph makes textual criticisms and explanations but the country name, city name, seal of the state of $\mathrm{Chu}$, and the county name, and also appendixes the seal of the state of Chu and the country name index. It is very convenient to use it.

There are large amounts as well about the research of vocabulary on Chu bamboo silk. The achievement of monograph "Shanghai Museum Collection of Chu Bamboo Slips in Warring States Period" Lost Literature Words Study (Qu bing, 2012) would be published in the form of lost literature vocabulary of Shanghai Museum.

Besides, the special book dictionary (Zhang Zaixin,2010) which compiles Bao Shan bamboo silk and Guo Dian banboo silk could be seen. Song Huaqiang (2010) collated and researched the Chu bamboo silk of Xincaige tomb, and make compilation of nearly more than ten monosyllabic words in Chinese Polysyllabic words.

In the aspect of hackling Qin bamboo silk vocabulary, even though there are still some deficiencies about the judgment of the word of the speech and the textual research and explanation of words meanings. But it does not affect seriously, because it has full range of words examples, rich sense of meanings and exhaustive description. In addition, there are also some achievements of LngGang bamboo silk, Zhou Jia Tai bamboo silk in Guan Ju, and Tianshui Fangmatan bamboo silk.

Moreover, there are a large number of papers make textual annotations in the aspect of characters and words of Qin bamboo silk, such as Li Fengjuan (2011).Li annotated five kinds of monosyllabic words and polysyllabic words from material of Qin bamboo slips, and there are nearly sixty-six monosyllabic words and polysyllabic words. And Xia Liya (2011) made the concentrating collation of Qin bamboo slips. Unfortunately, it is only directed against parts of Qin bamboo slips, and retained the content of majority, so the referential is low.

The paper (Liu Cuicui, 2010) takes the bronze inscription in Warring States period as the subject of study, and comprehensively hackles the vocabulary of the bronze inscription, and collects five kinds of the notional words of the inscription on bronze, such as nouns, verbs, adjectives, pronouns, and numeral. Therefore, it is of extraordinary 
significance for confirming a principle of monosyllabic words and polysyllabic words.

The research outcomes of interdisciplinary specialized vocabulary are still not enough. For instance, the monograph (Li Mingxiao, Zhao Jiuxiang, 2011) reorganizes the specialized vocabulary, such as unearthed legal literature of Warring States period.

\section{GRAMMAR STUDY}

Scholars have been make reviews and summary about the present of study on grammar of unearthed literature in Warring States period in recently. For instance, Chen Weiwu (1998),Zhou Shoujin(2004),Zhang Yujin(2011), and Yao zhengwu (2015), especially the study of Zhang Yujin (2011). According to the different kinds of corpus which have been used by researchers,Zhang reviewed it in four aspects as follow.

Firstly, the grammar study of Qin bamboo slips. Secondly, the grammar study of $\mathrm{Chu}$ bamboo silks.Thirdly, the grammar study of bronze inscriptions in Warring States period. Fourth, various grammar study of unearthed literature is in Warring States period. This work used most of the published literature materials of the Warring States to the Qin Dynasty. And it has made scientific analyses and conclusions in the issue about the era of various literatures, such as the age of formation, the age of transcription, and the age of tombs. Besides, it made an intensive study about function words (prepositions, conjunctions, auxiliary particles, modal particles and simultaneous words) of unearthed literature and handeddown Warring States literature based on facts. At last, according to the study situation, this work gave innovative reviews and evaluations about different kinds of unearthed literature.

There are most papers have been published to discuss the unearthed literature grammar in Warring States period in recent years, but few outcome monograph have been devoted to the study. The single doctor dissertations were more common, but from the research methods, there are still many deficiencies. For example, Xiong Changhua (2010) took a comprehensive grammar study of Dragon Gang Qin bamboo slips. It is consisted of morphology and syntax this two parts in the article written by Xiong. The division of function words is divided into adverbs, prepositions, conjunctions and auxiliary words in this article, and theories and methods are rather old, such as the judgment standard of word class and so on

And another example, the compilation and research of Xincaige Tomb Chu bamboo slips from She Ping (2010).It divides notional words in to nouns, verbs, adjectives, numeral, and pronouns this five kinds. However, the categories of verbs are divided into behavioral verbs, quasi stop verbs, perceptual verbs, state verbs, existential verbs and verbs of will, and to compare the first five categories, whether be a logical repetition, still worth discussing. But there remain have the merits wroth to be mentioned, for example, every polysyllabic words of the word frequency, determinant, and the explanation of words meanings are concise and brief.

\section{INTERCHANGEABLE WORDS COMPILATION}

With regard to study of interchangeable words, in Chinese ancient character circles, the research of interchangeable words from Wang Hui $(1993,2008)$ is most abundant. The range of era from Shang Dynasty to Qin Dynasty is involved, and it is impartial and credible of the classification. But, for the characters (mainly refers to the phonogram character) which were not recorded in later character books (rhyme dictionary), and whether be worth to discuss the issue of marking its ancient Chinese phonology. As far as the issue of labeling the tone, there are still many places worthy of discussion.

The study of interchangeable words in recently, there are many explanations and collations of monographs of Chu bamboo slips characters. And the kinds of corpus collections are limited to bamboo slips and silk book this two kinds, such as Liu Xinfang (2011), Bai Yulan (2012). And about the issue of era, the later monographs included bamboo slips and silk book materials after Qin Dynasty and Han dynasty, instead of a complete literature material of Warring States period. In addition, the Bai finishing method inherited interchangeable words in recent years could be seen, for example, Tang Hongzhi (2013) the postdoctoral report, its value or available for use in the revision of Bai.

The pioneers of this field are still scanty, however, there are still achievements deserve a treat, such as the paper The Construction of Ancient Silk Interchangeable Words Sound System and Database in The Warring States Period (Bai Yulan, 2014).And this project arrangement must to be based on the expansion and revise of In the Warring States Period and Ancient Books Borrowed Vocabulary Compilation. It is worthy to affirm with Bai's interchangeable words sound system as the method of making textual criticisms and explanations of Warring States characters and even unearthed literature. At the same time, it shows that its method and visual angle followed Dictionary of The Warring States Period - Text Sounds of The Warring States Period (He Linyi, 1998).

\section{COMPILATION OF CORPUSINDEXING TOOLS}

The index tools books mentioned here include unpublished papers, but it mainly comment published index tools books

In terms of material types, about the collation of bamboo silks corpus, there are indexes of Qin bamboo slips and Chu bamboo silks compiled word for word(Liu Zhiji,2012), (Zhang Xiancheng,2010,2013,2015), and it gets new interpretation results from scholars for other side in recent years. Furthermore, such as Wuhan University and Research Center (20112013) has published Chu Unearthed the Warring States Brochure Collection (I) (II). Comprehensive revision of Chu bamboo slips of literature such as Fourteen Kinds of Historical Records of Unearthed in Chu During The 
Warring States (Pan Meiyue, Lai Yixuan, 2012) may be more creative.

About the collation of ancient seal annotations and verbatim index, there are desirable results as well. For example, Zhou Ling carried out revisions and supplements about GuXi Assembly (古隹汇编), GuXi Wen Bian (古筀文编), etc. Zhou's monograph is bound to be a powerful booster to promote the study of ancient seal characters in the future, so this article with a huge expectation for the published of this monograph and other papers of Zhou Ling.

\section{CONCLUSION}

In summary, the academic circles have gained a bunch of achievements in the field of explanations and collations of Warring States unearthed literature materials, and vocabulary collections and language analysis, and so on. Especially in text editing, verbatim indexing tools books, difficult word explanation and thematic vocabulary consolidation, fruitful, comprehensive material in the future and lay a good foundation for the comprehensive the notional words of the Warring States period, and dictionary compilation.

In addition, the published Warring States literature materials are brilliant in recent years, for instance Warring States bamboo slips collected in Shanghai Museum, Warring States bamboo slips collected in Tsinghua University, Qin bamboo slips collected in Yuelu Academy, Liye Qin bamboo slips, and Qin bamboo slips collected in Peking University, etc. This new materials have been published one after another and related scholars continue in-depth research. Therefore, a pile of problems about have difficult word interpretation already been addressed.

Meanwhile, there are obvious limitations of some results. First, opinions about explanation of the text are varying, and some latest ideas could not be reflected in common Chinese reference books. Second, perhaps it could not play a great role in academic guidance, if authors of reference books still put difficult words as index, instead of absorbing new ideas. Third, study of the interchangeable words, ancient and modern words and other issues is still relatively weak. And system of initials and finals which summed up by handeddown rhythmic dictionary is conformed to realistic or not. Those issues still need to discuss. Fourth, the issue about how to eliminate the corpus which before the Warring Sates period from the grammar study of unearthed Qin literature in the Warring States period, still no attention has been paid yet. Even though some scholars have been aware that the scale of the problem, in the duration of the descriptive research on dynastic grammar, how to eliminate the principles and methods of the language before the Warring States period is still at the initial stage. Therefore, based on the current situation and existing limitations, we believe that new materials will emerge in the future, and new methods or new ideas will gradually improve the limitations of the existing problems.

\section{ACKNOWLEDGMENT}

This research was financially supported by the Project of Undergraduate Teaching Reform of Higher Education in Guangxi in 2016 (The reform and research of "practical teaching" of the Major of Teaching Chinese to Speakers of Other Languages on the Base of Personnel Training among Universities in Guangxi , having the project index number 2016JGA198). It was also supported by the project of Education and Teaching Reform of Guilin University of Electronic Technology in 2016 (Training and Practicing the Teaching and Research Ability of International Chinese Education Undergraduate Students in Micro-Lecture Times - taking Chinese Culture Curriculum as an Example, having the project index number JGB201634) and the project of the 'cultivation project of scientific research' of School of Foreign Language, Guilin University of Electronic Technology (Theory and Practice of Research Teaching among International Chinese Education Undergraduate Students-Taking Ancient Chinese I as an Example, having the project index number CS16028X). Thanks again.

\section{REFERENCES}

[1] Yujin Zhang.A Research on Function Words in Excavated Materials of the Warring States [M].Beijing: People press, 2011.

[2] Yujin Zhang. The Retrospect and the Prospect of A Research on Grammar in Excavated Materials of the Warring States [J]. Journal of South China Normal University (JCR Social Science Edition), 2007.

[3] Zhiji,Liu. Overview of ancient text interpretation [K]. Shanghai: Shanghai people's publishing house, 2011.

[4] Zongyi Rao, Zaiguo Xu.The book of chu zhuzhu in the warring states period [K]. Hefei: anhui university press, 2012

[5] Cuicui Liu.A study on the vocabulary of jinwen in the warring states period [D]. Master's thesis, east China normal university, 2010.

[6] Mingxiao Li.A study on the grammar of chu in the warring states period [M]. Wuhan: wuhan university press, 2010.

[7] Changhua Xiong. Study on the grammar of qinjian of longgang [D]. Master's thesis of southwest university, 2010.

[8] Shoujin Zhou A study on the study of ancient Chinese literature [M]. Beijing: Peking University press, 2005.

[9] Zhenwu Yao.The history of ancient Chinese grammar [M]. Shanghai: Shanghai ancient books press, 2015.

[10] Yanmei Zhang. The Retrospect and the Prospect of a Research on Pronouns in Excavated Materials of the Warring States[J]. International Conference on Contemporary Education, Social Sciences and Humanities (ICCESSH), 2016.

[11] YanmeiZhang. Pronouns Study in China-Taking a Research on Pronouns in Excavated Materials of Pre-Qin Dynasty as an Example[J]. International Conference on Education, Language, Art and Inter-cultural Communication (ICELAIC), 2016.

[12] Yanmei Zhang. The Present Situation and Existing Issues of Monographs Grammar Study of Excavated Documents - Taking Pronouns Study as an Example[J]. International Conference on Humanities and Social Science (HSS),2017. 